\section{High-Resolution Imaging with Atomic Force Microscopy}

\author{
Sergei Magonov
}

Veeco Instruments Inc., Santa Barbara, CA

The invention of scanning tunneling microscopy (STM) in 1982 [1] revolutionized surface analysis by providing atomic-scale surface imaging of conducting and semiconducting materials. Shortly after that, atomic force microscopy (AFM) was introduced as an accessory of STM for high-resolution imaging of surfaces independent of their conductivity [2]. Mechanical force interactions between a sharp tip placed at one end of a microfabricated cantilever and a sample surface were employed for imaging in this method. In the past decade, AFM has developed into a leading scanning probe technique applied in many fields of fundamental and industrial research. The progress of AFM has been made possible by implementation of an optical level detection scheme [3],
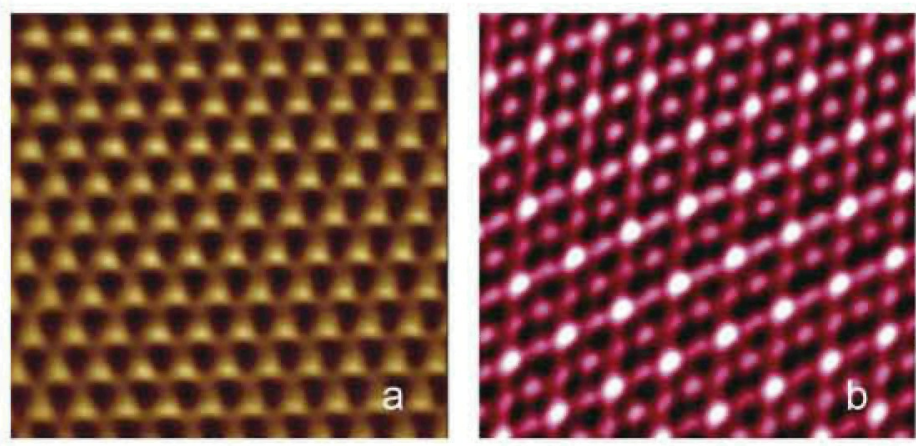

tion surface imaging, compositional mapping of multi-component materials, visualization of structures with different electric and magnetic properties, and measurements of local mechanical and thermal properties. AFM has become the characterization method that complements microscopic, diffraction, and other techniques. Many current AFM applications that provide new information for researchers in academia and industry are not necessarily related to imaging at small scales. As a result, visualization of nanometer scale structures offered by AFM has been largely overshadowed. However, due to increasing efforts in nanoscience and nanotechnology, interest in nanometer visualization has been revitalized. Several issues of high-resolution AFM imaging are discussed below.

AFM was introduced utilizing the contact mode technique, where the tip is in permanent contact with the surface during scanning. In this mode, images of crystal surfaces can show atomic-scale patterns identical to the crystallographic lattices [5]. The images of four different samples in figure 1 demonstrate this capability. The examples include surfaces of graphite (1a) and an-
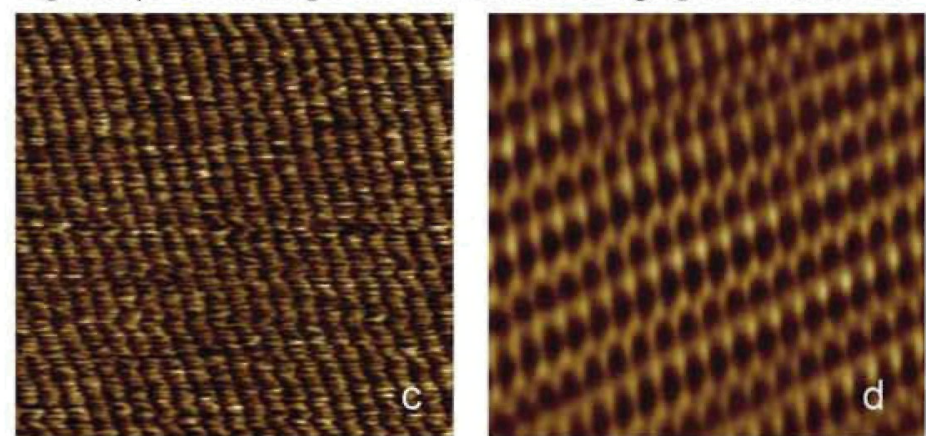

Figure 1. Contact mode AFM images of graphite (a), $\beta-\mathrm{Nb}_{3} \mathrm{I}_{8}(\mathrm{~b})$, polytetrafluoroethylene (c) and polydiacetylene crystal (d). 3 nm, $4.5 n m, 12.5 n m$, and $8.5 \mathrm{~nm}$ scans.

which allows precise measuring of the cantilever deflection caused by the tip-sample forces, by mass microfabrication of probes consisting of cantilevers, and by developments of oscillatory imaging modes, particularly, TappingMode ${ }^{\text {rut }}$ [4]. This mode is most broadly applied for measurements in a variety of environments (air, liquid, vacuum, etc.). All of these innovations have been put into practice in commercial instruments that have been installed in thousands of laboratories worldwide.

Exploring AFM capabilities, which was driven by the pioneering spirit of researchers towards high-resolution imaging and by the availability of advanced instruments, has led to a variety of applications, that are now well beyond the initially expected visualization of surface structures at atomic- and molecular-scale. At present, AFM is a multifunctional technique that is used for high-resolu-
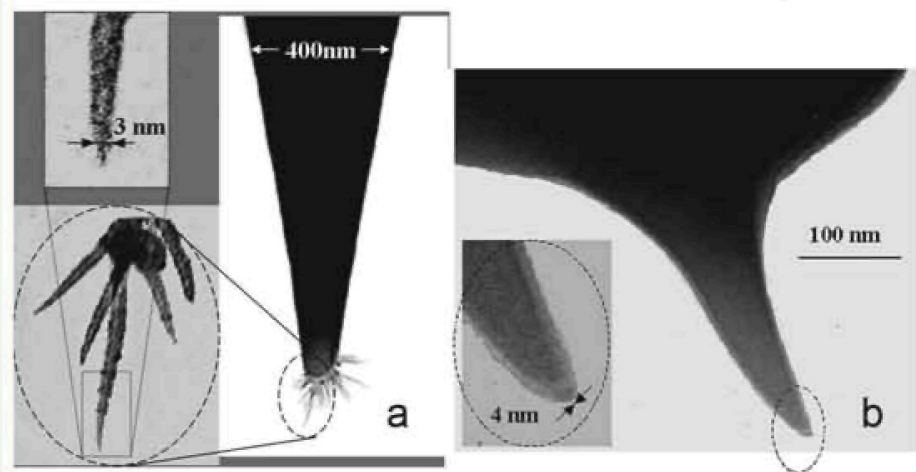

Figure 2. TEM micrographs of novel AFM probes. Probe with carbon spikes (a), and Mechanically sharpened diamond tip (b). other layered material, $\beta-\mathrm{Nb}_{3} \mathrm{I}_{8}(\mathrm{lb})$, as well as oriented chains of polytetrafluoroethylene film rubbed on glass (1c) and the surface of a polydiacetylene crystal (1d). The absence of atom-size defects in such images suggests that the tip contact area in this mode is larger than the atomic size. This does not exclude a possibility of lattice imaging based on periodic variations of normal and lateral forces that are experienced by a tip when it is moving along a periodic surface [6]. Lateral tip-sample forces that accompany scanning of surfaces with the tip in contact can be recorded for evaluation of surface friction. Unfortunately, lateral forces applied to soft samples might also induce a strong shearing deformation and sample damage. This limits the contact mode applicability to studies of polymers and biological objects.

The obstacles to AFM imaging of soft surfaces have been overcome with the development of TappingMode, where the tip contacts the sample surface at high frequency (tens and hundreds of $\mathrm{kHz}$ ), thus restricting sample damage. This mode has revolutionized AFM applications because a broad range of samples and materials of industrial importance have become accessible for studies at ambient conditions. The resolution of TappingMode is defined by the size of the tip-sample contact, which depends on the size of the tip's apex and tip-force. In this mode, the tip-sample forces are controlled by variations of the amplitude of the probe interacting with a sample. The use of the cantilever's frequency or phase, both of which are more sensitive to tip-force than is amplitude, for feedback in TappingMode, is not practical due to imaging instabilities at ambient conditions. The changes of the probe's frequency are more valuable 

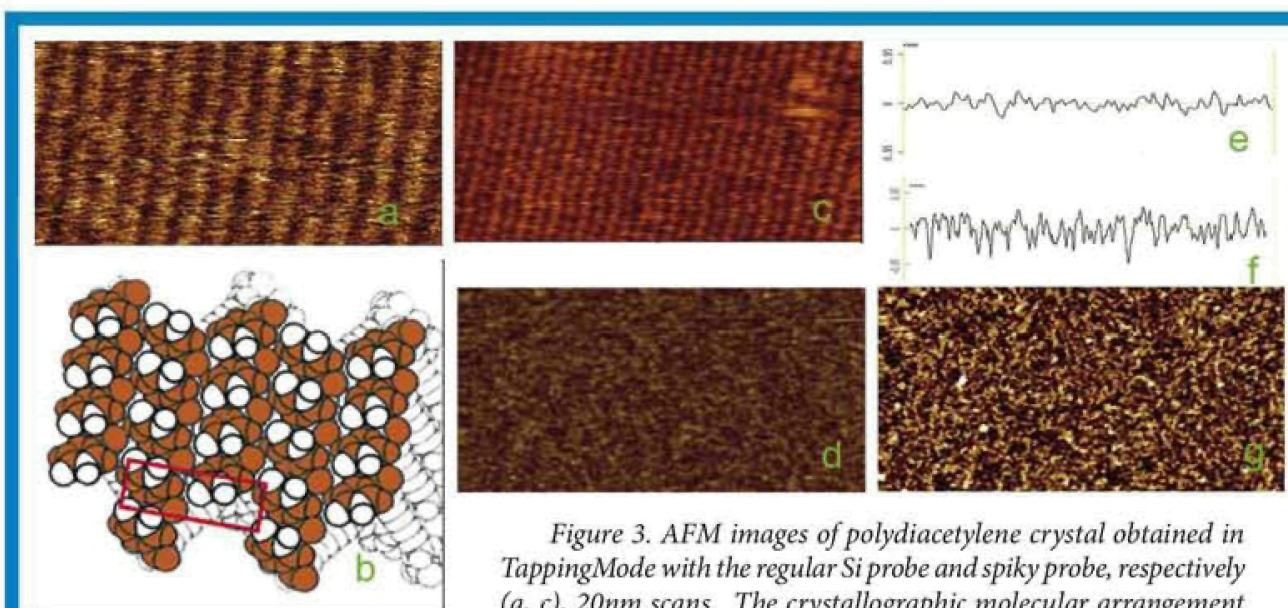

Figure 3. AFM images of polydiacetylene crystal obtained in TappingMode with the regular Si probe and spiky probe, respectively $(a, c), 20 \mathrm{~nm}$ scans. The crystallographic molecular arrangement of the surface of polydiacetylene (b). AFM images of Si wafer obtained in TappingMode with the regular Si probe and spiky probe, respectively $(d, g), 1 \mu \mathrm{m}$ scans. The height profile taken across images in $(d)$ and $(g)$, respectively $(e, f)$.

for feedback in another oscillatory mode - frequency modulation, which is applied to materials in UHV. In this environment, highprecision force control leads to a minimization of the tip-sample contact and imaging with true atomic-resolution [7]-although AFM applications in UHV are quite limited.

It is rather difficult to anticipate improvements of the force control in TappingMode, therefore, its imaging resolution might get better only when sharper tips become available. So far, most applications are made with etched Si probes, whose tip apex is around $10 \mathrm{~nm}$, and an image resolution that approaches $1 \mathrm{~nm}$. There are continuing efforts to design novel AFM probes. A possible use of carbon nanotubes as AFM probes is the focus of many researchers. Despite high expectations, there is no solid evidence so far that such probes provide better resolution than Si probes. Novel probes, in which carbon spikes with $\sim 1 \mathrm{~nm}$ ends are grown at the apex of the $\mathrm{Si}$ probes, figure $2 \mathrm{a}$, show better promise. These probes with spikes are produced in a batch process, yet the presence of multiple spikes limit their ease-of-use. Mechanically sharpened diamond tips [8] with a single extremity of a few $\mathrm{nm}$ in size (figure $2 \mathrm{~b}$ ), are also candidates for high-resolution probes, but they may be costly. Recently, the promise of higher resolution imaging of TappingMode with sharper probes has been proven [9]. This is shown in figure 3 , where images were obtained with a regular Si probe and a spiky probe. The crystal surface of polydiacetylene was chosen for this rigorous check. It is worth noting that the same surface had been examined earlier with contact mode (figure 1d), and the image corresponded well to the surface structure, which was reconstructed from the crystallographic data (figure $3 \mathrm{~b})$. This was not the case when the image was obtained in TappingMode with a Si probe (figure $3 a$ ). This image exhibits multiple strips, which are sep-
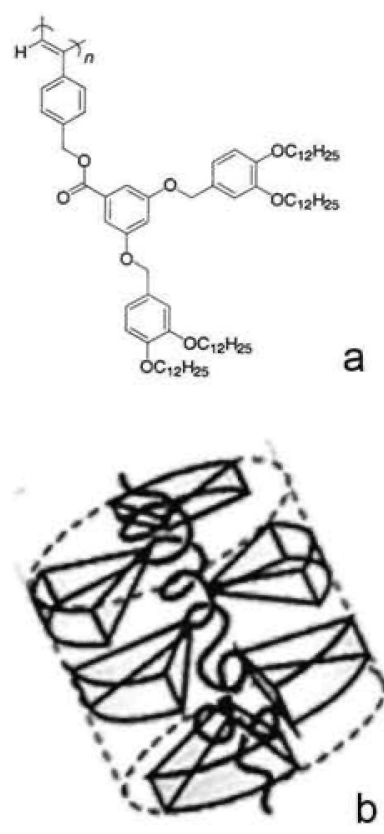

arated by $\sim 1.4 \mathrm{~nm}$ spacing, and each strip represents two unresolved molecular rows forming at the surface (compare figures 3a and $3 \mathrm{~b}$.) When the spiky probe was applied, an image with true molecular resolution was obtained, figure $3 c$. A well-resolved pattern with bright spots corresponding to individual molecular groups on the surface is seen in this figure. In addition, one can notice a molecular-scale defect, which is seen as a bright spot in the right-top of the image. High-resolution imaging with carbon spikes has also been proven in a study of a Si wafer, figures $3 \mathrm{~d}$ and $3 \mathrm{~g}$. It is obvious that the nanoscale roughness of the Si surface is much higher (over 2 times) in the image obtained with the sharper probe. This is also seen in the cross-section height profiles, figures $3 \mathrm{e}$ and $3 \mathrm{f}$. These results undoubtedly show capabilities of TappingMode imaging with resolution not possible with the etched Si probes. At present, such imaging is not trivial due to imperfections of the tip geometry and peculiarities related to the tip fragility. The delicate tip-sample engagement and careful scanning are needed to avoid spike damage.

High-resolution AFM imaging, which is enhanced with sharp probes, provides unique possibilities for visualization of individual macromolecules and their self-assemblies. In studies of single polymer chains on different substrates, the macromolecules of polyphenylacetylene [10] with mini-dendritic groups (figures $4 \mathrm{a}-$ c) were examined on a fracture surface of the bulk sample (figure $4 \mathrm{~d}$ ), and also on mica and graphite substrates, figures $4 \mathrm{e}$ and $4 \mathrm{f}$, respectively. The ordered chain layer was observed on the fracture surface, figure $4 \mathrm{~d}$. The distance between the chains measured in this
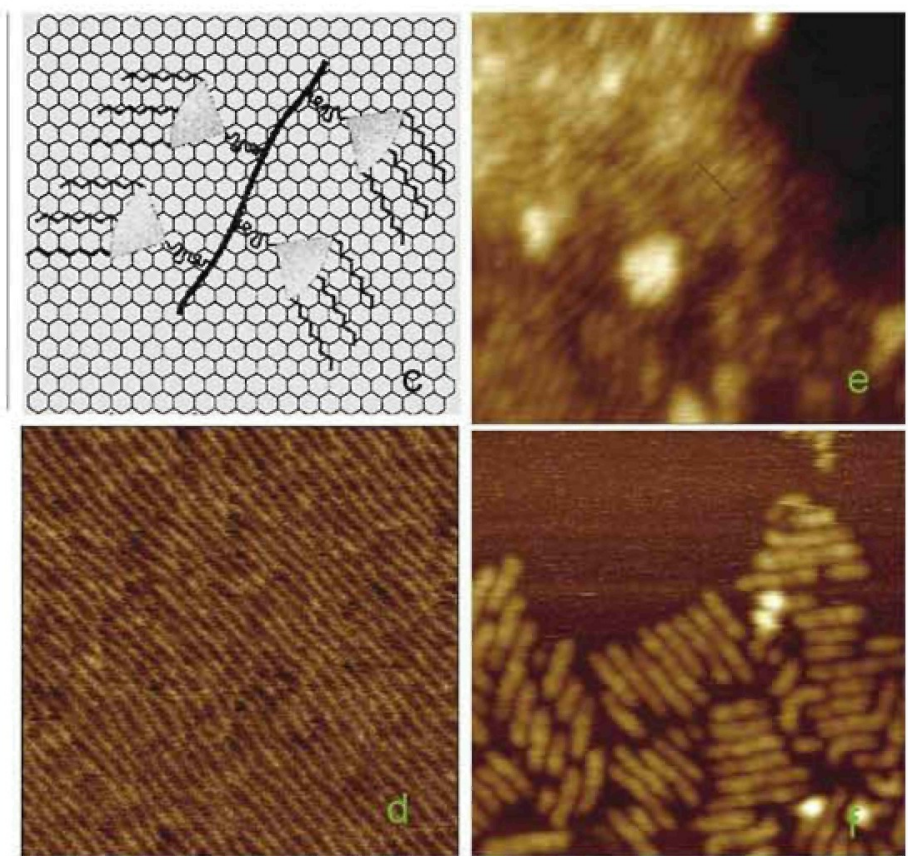

Figure 4. Chemical structure of polyphenylacetylene with mini-dendritic groups (a). A suggested shape of the polymer macromolecule with a diameter of $5 \mathrm{~nm}$ in bulk based on $X$-ray analysis. (b). A sketch demonstrating a possible epitaxy of the polymer chains on graphite (c). AFM images of the polyphenylacetylene with minidendritic groups on the fracture surface of the bulk polymer sample, on mica and on graphite, respectively ( $d$, $e, f) .150$ nm scans. 

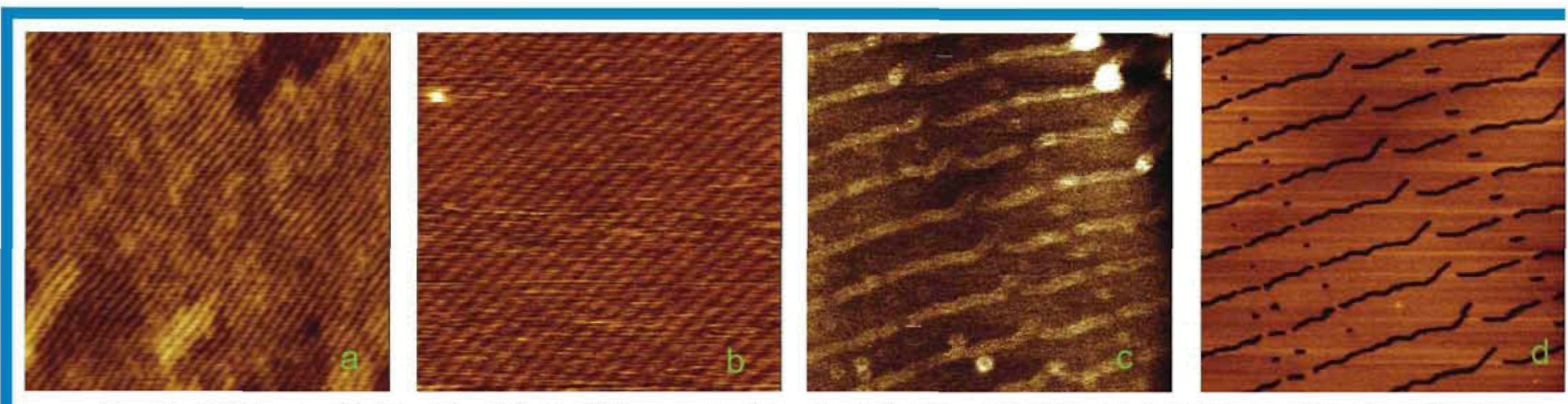

Figure 5. AFM images of $\mathrm{C}_{60} \mathrm{H}_{122}$ (a) and $\mathrm{C}_{36} \mathrm{H}_{74}$ (b) layers on graphite, respectively. 300nm and $150 \mathrm{~nm}$ scans. AFM images of $\mathrm{C}_{390} \mathrm{H}_{782}$ alkane layer on graphite $(c, d)$ that were obtained in light and hard tapping, respectively. $350 \mathrm{~nm}$ scans.

image $(4.7 \mathrm{~nm})$ corresponds to the macromolecule's diameter $(5.0$ $\mathrm{nm}$ ), which was determined by X-ray analysis of the bulk polymer. The same chain width was found in the image of the polymer aggregate on mica, figure $4 \mathrm{e}$. When the macromolecules were deposited on graphite, their epitaxy led to a chain alignment along the main crystallographic axis of the substrate, figure $4 \mathrm{f}$. The width of the macromolecules on graphite as measured in this image is wider $(6.5 \mathrm{~nm})$ than on mica; the height of the macromolecules is also reduced compared to that on mica. These observations suggest that the chains are flattened on graphite but they retain their cylindrical shape on mica. This finding confirms AFM value for conformation analysis of macromolecules on different substrates.

Visualization of the lamellar order of alkanes of different lengths on graphite is important for many reasons. AFM images of $\mathrm{C}_{36} \mathrm{H}_{74}$ and $\mathrm{C}_{60} \mathrm{H}_{122}$ layers (figures 5a-b) show regular lamellar aggregates with spacings of $4.6 \mathrm{~nm}$ and $7.4 \mathrm{~nm}$, which correspond to the molecules' length in the extended all-trans conformation. These images can help to calibrate the piezoelectric actuators - scanners - that are used in AFM instruments. This is important because man-made calibration samples for this scale are not available. The fact that the lamella width in image of alkanes are often visualized via an alternation of bright and dark linear features have very important consequences for understanding AFM images of alkanes and polyethylene. The reversible variations of the height images of ultra-long alkane $\mathrm{C}_{390} \mathrm{H}_{782}$ layers, figures $5 \mathrm{c}$-d, are observed when tip-sample forces are varied from low tapping to hard tapping, respectively [11]. At light tapping, the height image, figure $5 \mathrm{c}$, represents the surface topography, therefore, the sites of end - $\mathrm{CH}_{3}$ groups of the alkane, which are bulkier than $-\mathrm{CH}_{2}$ - groups, are seen elevated. When the tip-force increases, the tip penetrates through the mobile $-\mathrm{CH}_{3}$ regions, which are seen as depressed (darker contrast). Therefore, the contrast variations indicate the interlamellar locations. High-contrast of the regions helps visualization of structural defects and their dynamics in alkane and polyethylene layers on different substrates and at various temperatures [12]. This is also an example of AFM compositional mapping, which differentiates individual components of heterogeneous samples based on the differences of their mechanical properties.

In summary, high-resolution visualization of surface structures with AFM remains one of the most attractive features of this technique. It has challenges related to the use of novel sharp probes in air and other environments. But, it has potential for better understanding of the motifs of molecular self-organization.

\section{References}

1. G. Binnig, H. Rohrer, Ch. Gerber and E. Weibel, Phys. Rev. Lett. 49 (1982), 57.

2. G. Binnig, C. Quate and Ch. Gerber, Phys. Rev. Lett. 56 (1986), 930.

3. S. Alexander, L. Hellemans, O. Marti, J. Schneir, V. Elings, P. K. Hansma, M. Longmire and J. Gurley, J. Appl. Phys. 65 (1989), 164.

4. Q. Zhong, D. Innis, K. Kjoller and V. B. Elings Surf. Sci. Let. 290 (1993) L688

5. S. N. Magonov and M. $=$ H. Whangbo, Surface Analysis with STM and AFM, VCH, Weinheim (1996)

6. U. Landman, W. D. Luedke and A. Nitzan, Surf. Sci. Lett. 10 (1989), L177.

7. F. J. Giessibl, Science 267 (1995), 68.

8. The diamond probes are made by B. Mesa, MicroStar Technology, Huntsville, TX, USA, www.microstartech.com.

9. D. Klinov and S. Magonov, Appl. Phys. Lett. 84 (2004), 2697.

10. Sample is courtesy of Prof. V. Percec (UPenn, Philadelphia)

11. Magonov S. N., "AFM in Analysis of Polymers" Encyclopedia of Analytical Chemistry, (R. A. Meyers, Ed.), pp. 7432-7491, John Willey \& Sons Ltd, Chichester, 2000 .

12. Magonov S. N., Yerina N. A., G. Ungar and D. Ivanov, in preparation.

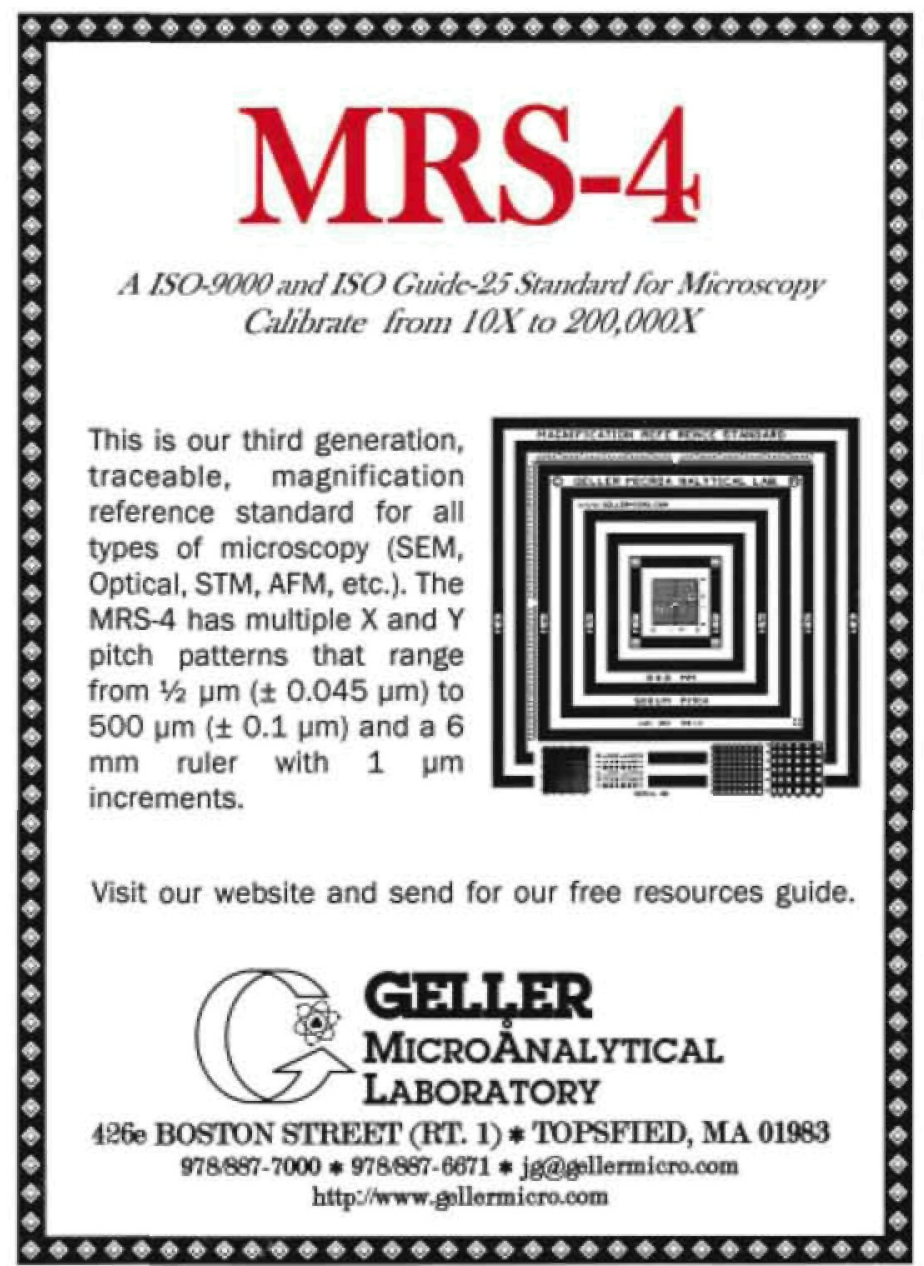

